\title{
Structural and Surface Morphology Analysis of Copper Phthalocyanine Thin Film Prepared by Pulsed Laser Deposition and Thermal Evaporation Techniques
}

\author{
Mohammed T. Hussein, Kadhim A. Aadim, Eman K. Hassan \\ Department of Physics, College of Science, University of Baghdad, Baghdad, Iraq \\ Email: Mohammedtake@gmail.com, Kadhim_adem@yahoo.com,Eman_kahreem@yahoo.com
}

Received 24 February 2016; accepted 16 April 2016; published 19 April 2016

Copyright (C) 2016 by authors and Scientific Research Publishing Inc.

This work is licensed under the Creative Commons Attribution International License (CC BY). http://creativecommons.org/licenses/by/4.0/

cC) (i) Open Access

\begin{abstract}
In the present paper, Copper Phthalocyanine (CuPc) thin films were deposited on glass and silicon substrate by thermal evaporation and pulsed laser deposition (PLD) methods. CuPc thin films prepared at different annealing temperatures $(298,323,348,373,423 \mathrm{~K})$ respectively. The structure and surface morphology of CuPc in powder and thin films forms prepared by two methods were studied using Energy dispersive X-ray (EDX), X-ray florescence (XRF), X-ray diffraction (XRD), Atomic force microscope (AFM), and Scanning electron microscope (SEM). It showed that there was a change and enhancement in the crystallinity and surface morphology due to change in the annealing temperature $\left(T_{a}\right)$. The purpose of our work is to find the optimal temperature for which the film produces best structural properties for CuPc thin film to produce organic field effect transistor. Analysis of X-ray diffraction patterns of CuPc in powder form showed that it had an $\alpha$-polycrystalline phase with monoclinic structure, with preferentially oriented (100) plane transform to $\beta$-single crystalline morestable structure at different annealing temperatures.
\end{abstract}

\section{Keywords}

Organic CuPc, Pulse Laser Deposition, XRD, EDX, AFM, Morphology

\section{Introduction}

The materials considered of most importance are the phthalocyanines (Pc) in the organic dyes and pigments. Phthalocyanine is a generally P-type semiconductor, thermally stable and can easily be sublimated as thin films 
with high quality. Phthalocyanine has been found application as semiconducting devices, organic field effect transistor OFET [1], organic humidity sensors [2], photovoltaic cells, rectifying devices, liquid crystal displays and gas sensors. The electrical, optical and structural properties of Pc thin films are dependent on various parameters such as evaporation rate, substrate temperature and annealing temperature [3].

Phthalocyanine is found at least two different crystalline phases i.e. $\alpha$ - and $\beta$-forms [4] [5]. It was reported that the $\beta$-form is a monoclinic crystal, more stable phase and may be thermally derived from the $\alpha$-form [6]. Metal free Pcs contain two hydrogen atoms in the center of molecule as shown in Figure 1. In order to increase the mobility of $\pi$-electrons, the two hydrogen atoms were replacing by a single metal atom.

Metal-substituted and metal free phthalocyanine are readily used as active layer in several applications such as gas sensors, photocopacitive and photoresistive detectors, Organic thin film Transistors (OTFT) [7], colour filters and organic laser materials. Among the various metal-substituted phthalocyanine, Copper phthalocyanine is one of the metal phthalocyanines. Figure 1 shows the molecular structure of Pc and CuPc. CuPc has an excellent chemical and thermal stability at ambient atmosphere and has applications to chemical sensors [8] and organic solar cells [9].

Pulsed laser deposition (PLD) has become a widely used deposition technique for thin film growth. PLD presents several advantages with respect to other deposition techniques. In fact, due to the high energetic content of the ejected species, it allows low temperature deposition process. Moreover, its ability to congruently transfer the stoichiometry from the target to the film allows the growth of complex materials. The technique is based on the vaporization process induced by focusing a high energy pulsed laser on the surface of the material. When the energy laser density is higher than a threshold value, which depends both on the material and the laser wavelength, a stream of atoms, molecules, and clusters are ejected from the target surface. Such a stream, known as a plume and being also composed of excited neutral and ionized species, emits radiation [10].

In the present work, the structural properties of CuPc thin films prepared by PLD and thermal evaporation methods at different annealing temperatures were studied. Annealing process is a broadly employed method to enhance the quality of the crystal and exploit structural defects in the material. During thermal annealing, the morphology and structural properties of the material change [11]-[13]. The purpose of our work is to find the optimal temperature for which the film produces best structural properties for CuPc thin film to produce organic field effect transistor.

\section{Experimental}

The CuPc blue pigment, with the molecular formula $\left(\mathrm{C}_{32} \mathrm{H}_{16} \mathrm{~N}_{8} \mathrm{Cu}\right)$ powder used in this work was obtained from sigma Aldrich chemical company (purity, 99\%). The powder is blue in color and easily soluble in organic solvent such as toluene, ethanol, etc. and was used as the source material for thermal evaporation and pulsed laser deposition techniques. The powder was pressed under a 5 tons press to form a target with $9 \mathrm{~mm}$ diameter and $5 \mathrm{~mm}$ thickness. The obtained target was as dense and homogenous as possible to ensure a good quality of the deposit.

Before starting the thermal deposition, the substrate was cleaned in an ultrasonic bath for 10 min using acetone, followed by rinsing in distilled water. The substrate was dried in open air in a cleaned room. A molybdenum boat was used as a heating source. Thin films of CuPc, were deposited by vacuum evaporation technique

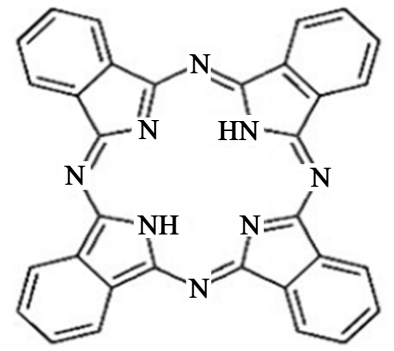

(a)

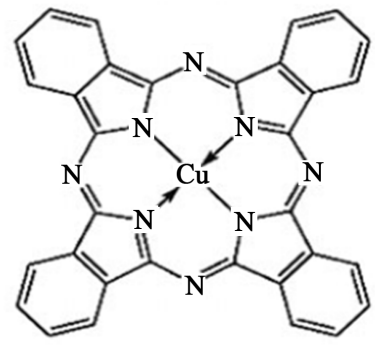

(b)

Figure 1. (a) Metal free phthalocyanine molecule, the central cavity contains two hydrogen atoms while in the (b) copper phthalocyanine molecule; the central cavity contains a copper atom. $\mathrm{N}$ is nitrogen, $\mathrm{H}$ is hydrogen and $\mathrm{Cu}$ is copper. 
on thoroughly cleaned substrate using Edwards 306 system. During deposition, the pressure in the vacuum chamber was kept constant at about $10-5 \mathrm{mbar}$, Also the substrate are placed at a distance of $15 \mathrm{~cm}$ from the source. Annealing of the prepared films was carried out at different temperatures $(298,323,348,373,423 \mathrm{~K})$ for one hour.

PLD was carried out inside a vacuum chamber in (10 - 3 torr). The focused Nd:YAG SHG Q-switching laser beam incident on the target surface deposited an angle of $45^{\circ}$. The films were deposited on glass and silicon wafer with rate of deposition equal to $11 \mathrm{~nm} / \mathrm{sec}$. the deposition was carried out a Q-switched Nd:YAG laser with a wavelength $1064 \mathrm{~nm}$ (pulse width $10 \mathrm{nsec}$ and repetition frequency 6Hz). Figure 2 shows a typical set-up for PLD system.

Structural analysis of the films was done by X-ray diffractometer (XRD) using (XRD-6000) supplied by SHIMADZU. The surface morphology of the CuPc films prepared by PLD and thermal evaporation methods are investigated by means of atomic force microscope (AFM) CSPM AA3000 AFM supply by Angstrom Company. AFM probes the surface of a sample with a sharp tip from Aluminum Nitrate (Al3N), a couple of microns long often less than $100 \AA$ in diameter. The tip is located at the free end of a cantilever, which is 100 to $200 \mu \mathrm{m}$ long. The forces between the tip and sample surface cause the cantilever to bend or deflect. A detector measures the cantilever deflection as tip is scanned over the sample or the sample is scanned under the tip. The measured cantilever deflection allows a computer to generate a map or surface topography. Scanning Electron Microscope studies were used to determine the size distribution of nanoparticles, structure and the shape using VEGA3 TESCAN, mode SE from TESCAN ORSAY HOLDING, a.s., Czech Republic, where high-resolution images of the surface of a sample is acquired. The microscope works by measures the secondary electrons from the sample. SEM is capable of magnifying images up to 200,000 times.

The lattice parameters of the films were calculated using the Bragg's formula:

$$
2 d \sin \theta=n \lambda
$$

The grain size of the crystallites was calculated from the XRD using Scherrer's relation:

$$
D=k \lambda /(\mathrm{FWHM}) \cos \theta
$$

where $k=0.94$ is a constant, $\lambda$ the wavelength of $\mathrm{X}$-ray $(\lambda=1.54 \AA$ ) of $\mathrm{Cu}-\mathrm{K} \alpha$ transition, (FWHM) the full width at half maximum and $\theta$ the diffraction angle. Scanning Electron Microscope studied was used to determine the nanoparticles distributions and nanoparticles size. $\beta$ angle and lattice parameters $(a),(b)$ and $(c)$ for monoclinic crystal can be calculated from X-ray d-spacing's according to equations:

$$
\operatorname{Sin}_{(\theta)}^{2}=\frac{\lambda^{2}}{4 \operatorname{Sin}_{(\beta)}^{2}}\left(\frac{h^{2}}{a^{2}}+\frac{k^{2} \operatorname{Sin}_{(\beta)}^{2}}{b^{2}}+\frac{l^{2}}{c^{2}}+\frac{2 h l \operatorname{Cos}(\beta)}{a c}\right)
$$

where $h, k$, and $l$ refer to the Miller indices of individual reflections and $\beta$ is the angle between $a$ and $c$.

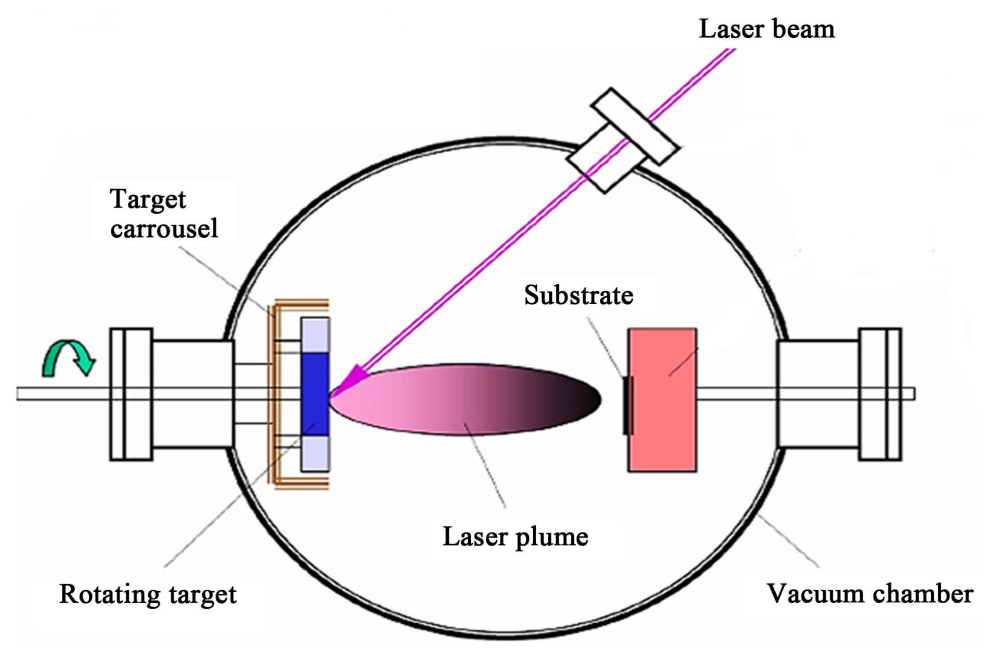

Figure 2. Schematic diagram of a typical Pulsed laser deposition (PLD) set-up. 
Analysis of Energy Dispersive X-ray (EDX) by Bruker Nano Gmbh, Germany SEM, using to show the purity and element percentage contain in the thin film. X-ray florescence was used to study the CuPc transition line.

The thickness of the films was measured using laser interferometer technique. The thickness of the film determined by this procedure equals to $500 \mathrm{~nm}$.

\section{Results and Discussions}

\subsection{EDX \& XRF Studies}

The chemical composition analysis was carried out on CuPc $\left(\mathrm{C}_{32} \mathrm{H}_{16} \mathrm{~N}_{8} \mathrm{Cu}\right)$ thin film sample by energy dispersive X-ray (EDX) analysis. Figure 3 shows the representative (EDX) pattern of $\mathrm{C}_{0.5} \mathrm{~N}_{0.5} \mathrm{Cu}_{0.5}$ thin film which confirms the formation of C:H:N:Cu presence of Carbon, Hydrogen, Nitrogen and Copper. An intense peak for Carbon was found in the spectrum due to the chemical stoichiometry composition $\left(\mathrm{C}_{32} \mathrm{H}_{16} \mathrm{~N}_{8} \mathrm{Cu}\right)$.

The CuPc molecule was studied by using XRF as illustrated in Figure 4. This figure indicated that the k-series of copper consist of an intense $\mathrm{k}_{\alpha}$ line and weak $\mathrm{k}_{\beta}$ and $\mathrm{k}_{\gamma}$ radiation, listed in the order of decreasing wavelength (1.542, 1.393, 0.775) A respectively and of increasing energy $(8.04,8.9,16) \mathrm{keV}$ respectively. These peaks gave an indication that the qualitative of the $\mathrm{Cu}$ present in the chemical compound prepared of CuPc.

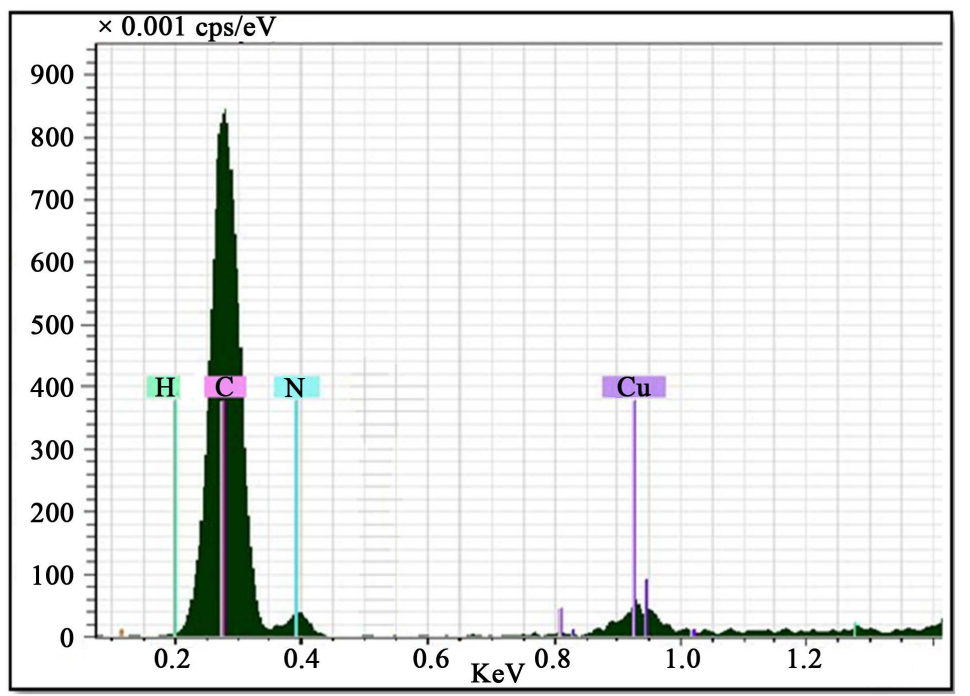

Figure 3. The EDX analysis of CuPc.

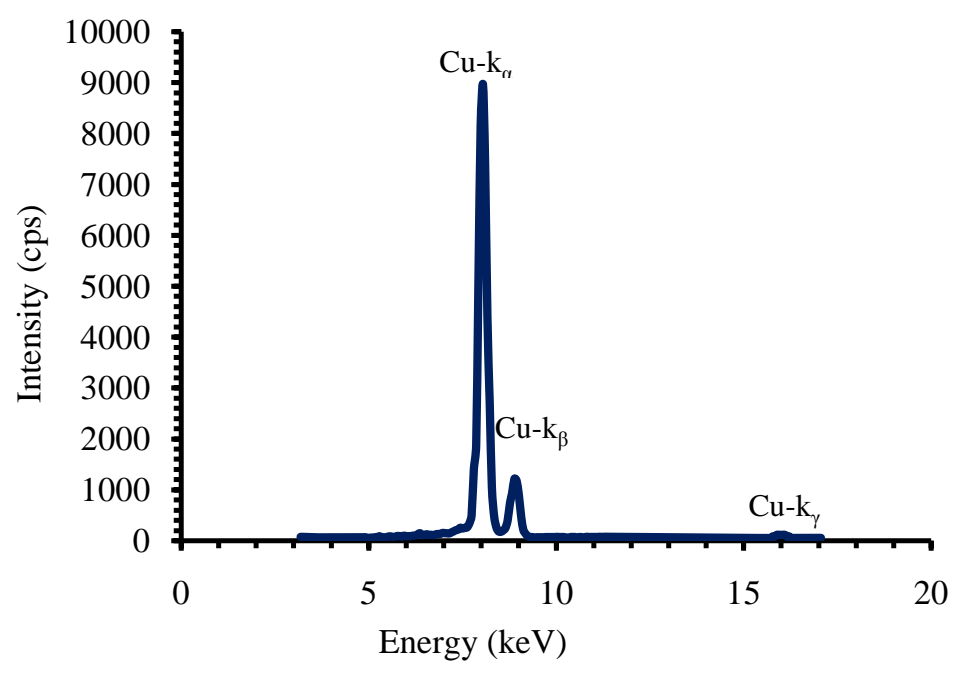

Figure 4. The spectrum of X-ray fluorescence for CuPc. 


\subsection{X-Ray Studies}

An X-ray diffraction of CuPc powder used as the source material for sublimation is shown in Figure 5(a). lattice spacing $d_{h k l}$ were calculated using Bragg's equation together with Miller indices and the calculated values of $d_{\mathrm{hkl}}$ are compared with the corresponding values given for $\beta$-phase with monoclinic structure in the ICDD card no. 11-0893 of $\beta$-CuPc. Table 1 shows the structure parameters of CuPc powder.

The preferential orientation is found to be in the (100) direction at $2 \theta=7.0947^{\circ}$ and that it has an $\alpha$-crystalline phase with monoclinic system with lattice constants $\mathrm{a}=1.468 \mathrm{~nm}, \mathrm{~b}=0.498 \mathrm{~nm}, \mathrm{c}=1.960 \mathrm{~nm}$ and $\beta=121.5^{\circ}$. The close agreement showed that the powder is in the monoclinic structure as reported for other phthalocyanine [4]. X-ray diffraction pattern of Si wafer n-type is shown in Figure 5(b). Peak at $2 \theta=28.4954^{\circ}$ showing the single crystalline nature of the wafer at (111) direction. Figure 5(c) shows the X-ray diffraction pattern of CuPc thin film on Si substrate deposited by thermal evaporation technique. From this figure we can obtained two special peaks one at $2 \theta=7.0540^{\circ}$ which corresponded to the (100) diffraction peak of the $\beta$-phase illustrated the highest peak of the CuPc crystal and the another peak at $2 \theta=28.8129^{\circ}$ which corresponded to the (111) diffraction peak of silicon. The XRD patterns of CuPc thin films deposited onto silicon at room temperature and annealing temperature (323, 348, 373, $423 \mathrm{~K}$ ) are shown in Figure 5(d). The XRD pattern shows the existence of a crystalline peak at $2 \theta=7.0540^{\circ}$ which corresponded to the (100) diffraction peak of the $\beta$-phase more stable of CuPc crystal. This is the environmentally stable crystal form at the annealing temperature lower than 423 K [14]. The intensity of (100) plane has increase for temp (323, 348, 373) K which means better arrangement and more crysttalinity This mean that the crystallization of the films is a function of annealing temperatures and decreased for $423 \mathrm{~K}$ which may be because of phase changing). The observed crystalline peak at (100) direction indicates the possibility to achieve high mobility due to the $\pi-\pi^{*}$ orbital staking. Table 2 shows the structure parameters of CuPc films. The full width at half maximum (FWHM) intensity of preferential orientation reduced with increasing annealing temperatures, which represents better lattice quality [15], and the crystallite size was estimated from Scherrer's equation [16].

From Table 2, the results show an increase in grain size with increasing annealing temperatures $T_{a}$ for (323, $348,373) \mathrm{K}$ due to the regrowth of phthalocyanine. This means that the crystallization of the films is a function of annealing temperature $T_{a}$ and decrease at $423 \mathrm{~K}$ because of phase changing. The effect of annealing temperatures on the crystallite size is shown in Figure 6, as observed, at annealing temperature $>423 \mathrm{~K}$, the grain size increases markedly with annealing temperature but at $423 \mathrm{~K}$ the grain size decreases, and this is agreement with other literatures [4].

Table 1. This table shows the structure parameters of CuPc powder.

\begin{tabular}{cccc}
\hline hkl & $2 \theta$ (degree) & Intensity (a.u.) & d $(\AA)$ \\
\hline$(100)$ & 7.0947 & 5750 & 12.44962 \\
$(\overline{1} 02)$ & 9.2099 & 3996 & 9.59456 \\
$(002)$ & 10.5720 & 492 & 8.36126 \\
$(\overline{2} 02)$ & 12.5023 & 782 & 7.07432 \\
$(200)$ & 14.0727 & 177 & 6.28821 \\
$(\overline{3} 02)$ & 18.1172 & 1156 & 4.89252 \\
$(011)$ & 18.5186 & 870 & 4.78736 \\
$(\overline{2} 11)$ & 21.2857 & 304 & 4.17085 \\
$(\overline{1} 13)$ & 22.9636 & 279 & 3.86975 \\
$(112)$ & 23.6641 & 1655 & 3.75676 \\
$(\overline{3} 11)$ & 26.0920 & 929 & 3.41243 \\
$(014)$ & 27.9034 & 541 & 3.19489 \\
$(311)$ & 30.3406 & 791 & 2.94357 \\
\hline
\end{tabular}




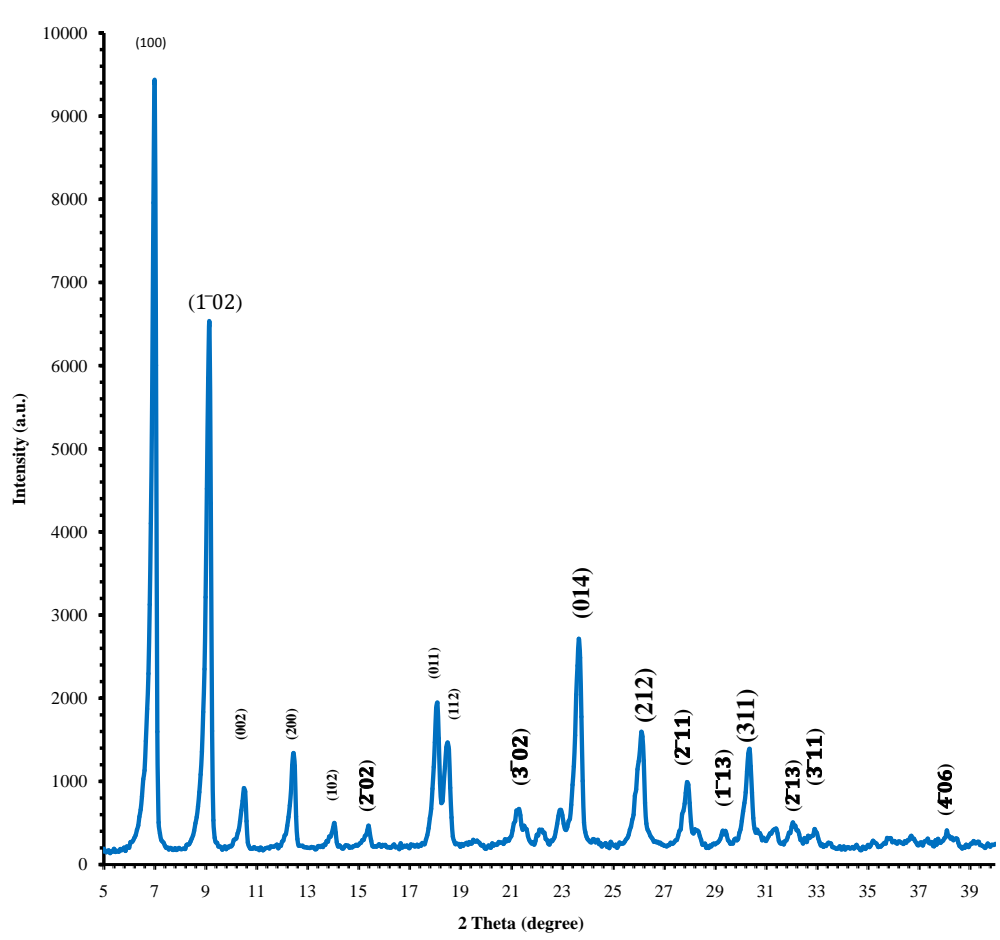

(a)

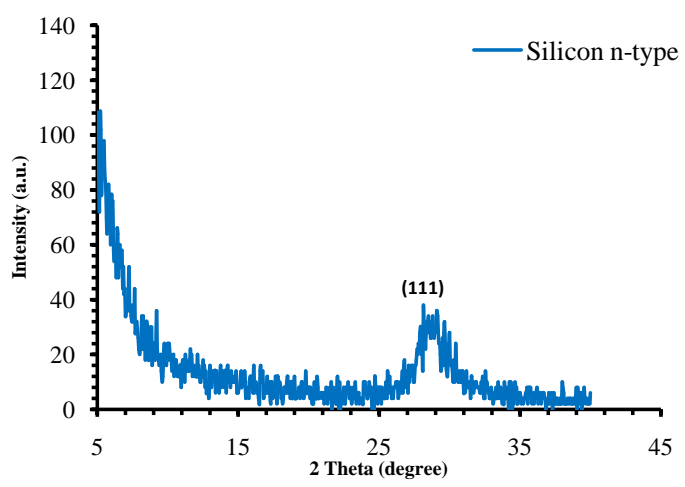

(b)

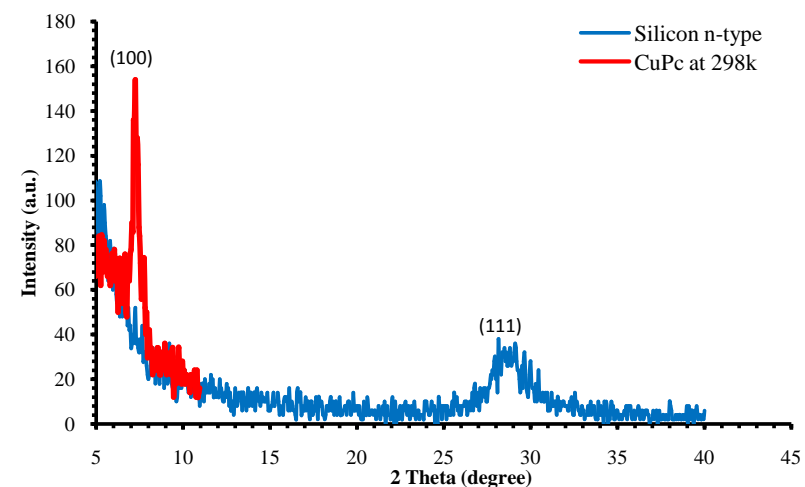

(c)

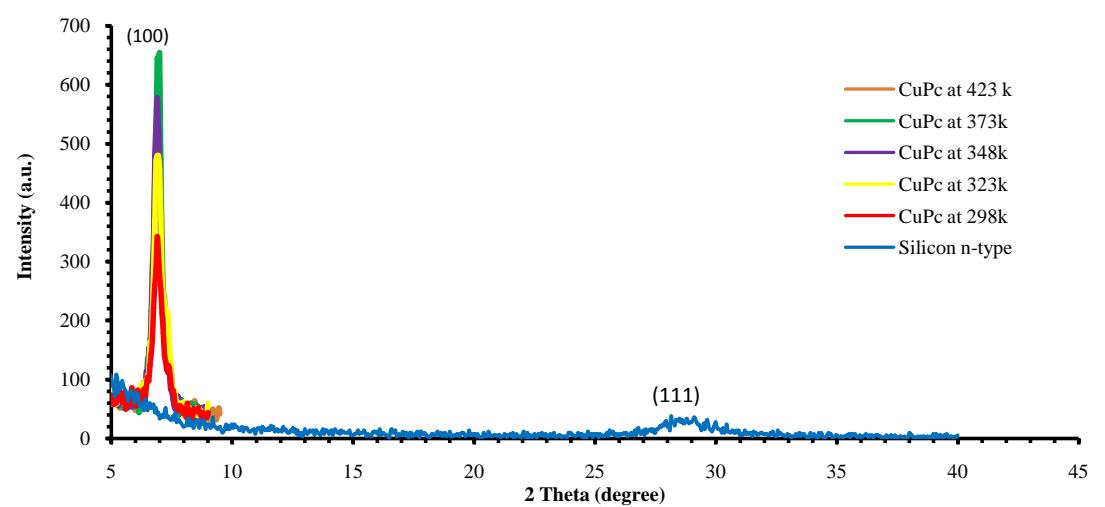

(d)

Figure 5. (a) The XRD spectrum for CuPc Powder; (b) The XRD pattern of Silicon wafer n-type; (c) XRD pattern of CuPc thin film on Silicon substrate at room temperature; (d) XRD pattern of CuPc thin film on Silicon substrate at room temperature. 


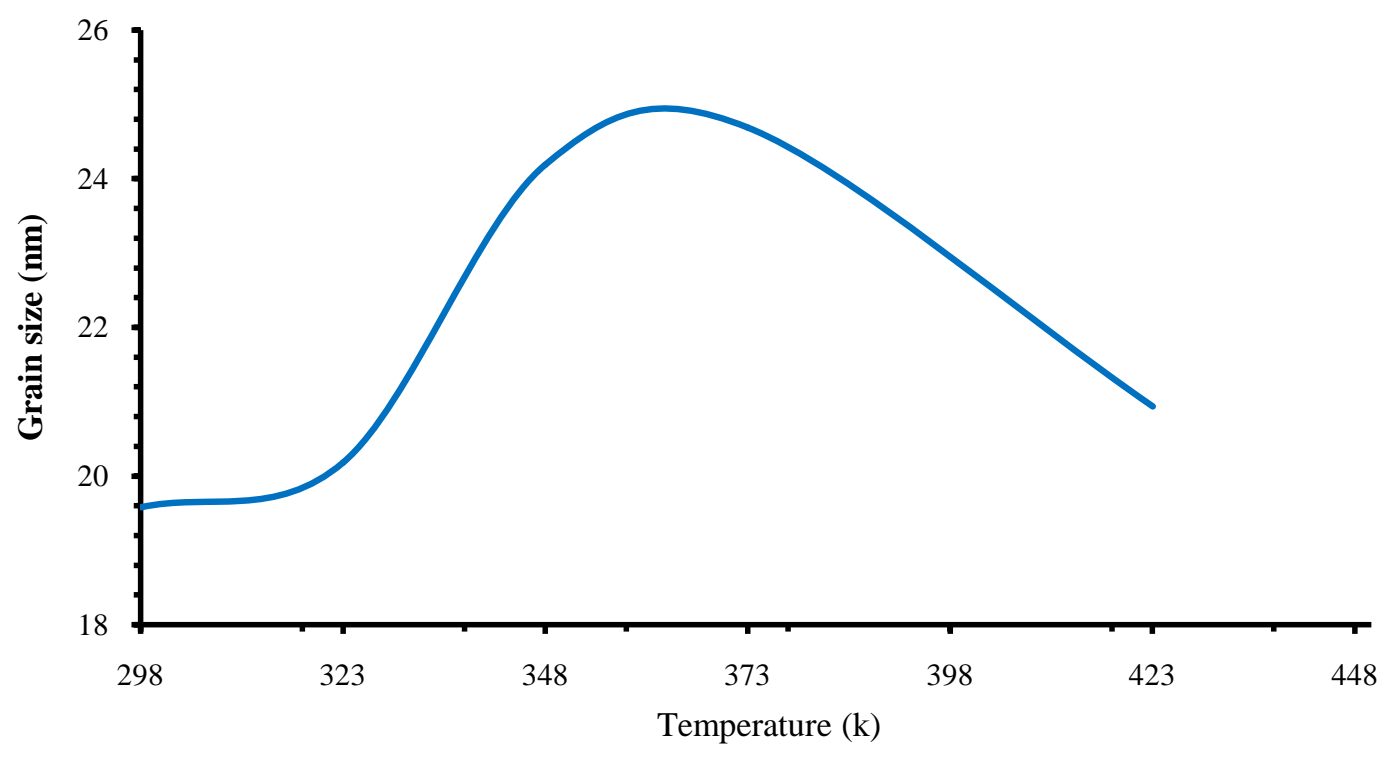

Figure 6. The effect of annealing temperatures on the crystallite size.

Table 2. This table shows the structure parameters of CuPc thin films using thermal evaporation technique.

\begin{tabular}{cccccc}
\hline $\mathrm{T}_{\mathrm{a}}(\mathrm{K})$ & $(\mathrm{hkl})$ & $2 \theta$ (degree) & $\mathrm{d}(\AA)$ & FWHM (degree) & $\mathrm{D}(\mathrm{nm})$ \\
\hline 298 & 100 & 7.0540 & 12.52136 & 0.42470 & 19.58 \\
323 & 100 & 7.0638 & 12.50401 & 0.41210 & 20.18 \\
348 & 100 & 7.0238 & 12.57513 & 0.34370 & 24.19 \\
373 & 100 & 7.0563 & 12.51728 & 0.33670 & 24.69 \\
423 & 100 & 7.0758 & 12.48283 & 0.39710 & 20.94 \\
\hline
\end{tabular}

Figure 7 shows the X-ray diffraction pattern of CuPc thin film on Si substrate deposited by PLD technique at different shoots. From this figure we can obtain special peaks at $2 \theta=7.1014^{\circ}, 7.1357^{\circ}$ which corresponded to the (100) diffraction peak of the $\beta$-phase of the CuPc crystal for 150 and 400 shoots, respectively and the another peak at $2 \theta=28.8129^{\circ}$ which corresponded to the (111) diffraction peak of silicon. Table 3 shows the structure parameters of CuPc films prepared using PLD technique.

From Table 3, the results show decrease in grain size with increasing no. of shoots due to the regrowth of phthalocyanine.

\subsection{AFM Studies}

In order to get more information on the structure of the deposited films prepared by thermal evaporation and pulsed laser deposition methods, the samples were examined using Atomic Force Microscope (AFM). Surface morphology of CuPc thin films prepared by thermal evaporation method and deposited on glass substrates are shown in Figure 8 and Figure 9, which annealed at different temperatures (298, 323, 348, 373, 423 K). It shows that the morphology of the CuPc thin film has large number of grain size and are homogeneously distributed, which indicates the crystalline nature of the film. It is obvious because the film were prepared at different annealing temperatures due to the heating grain growth will takes place which gives the crysttalinity and good surface morphology [11]. These results are in good agreement with XRD characteristics. The crystal morphology and molecular orientation change with annealing temperature.

These structural characterizations confirm that the annealing temperature allows us to control the structural organization of CuPc molecules in their solid state, from which a large improvement of the properties can be expected. Table 4 shows a fluctuation in grain size with increasing the annealing temperatures $T_{a}$. The root 


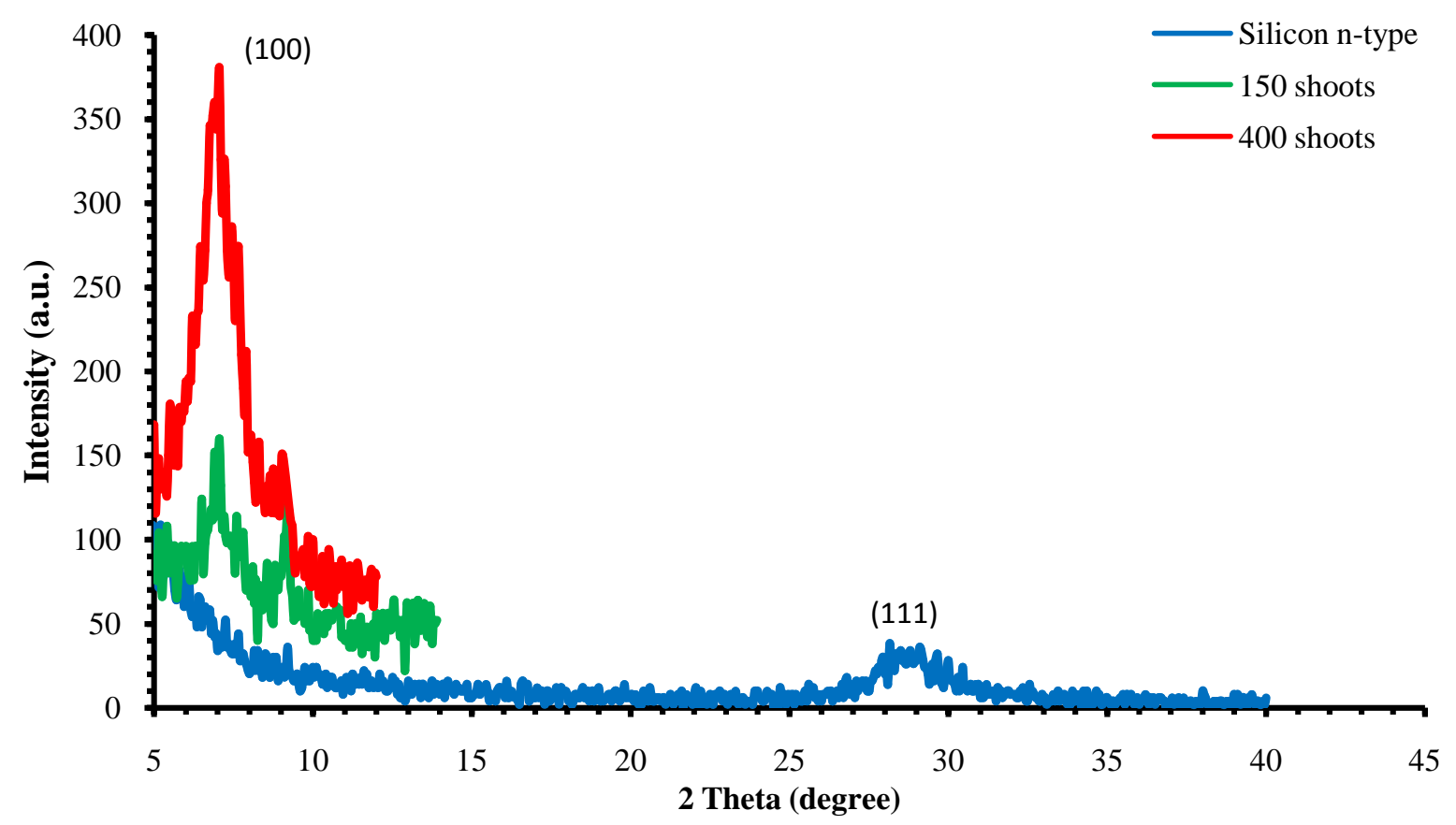

Figure 7. The XRD patterns of CuPc thin films deposited on silicon substrate at room temperature using PLD technique.
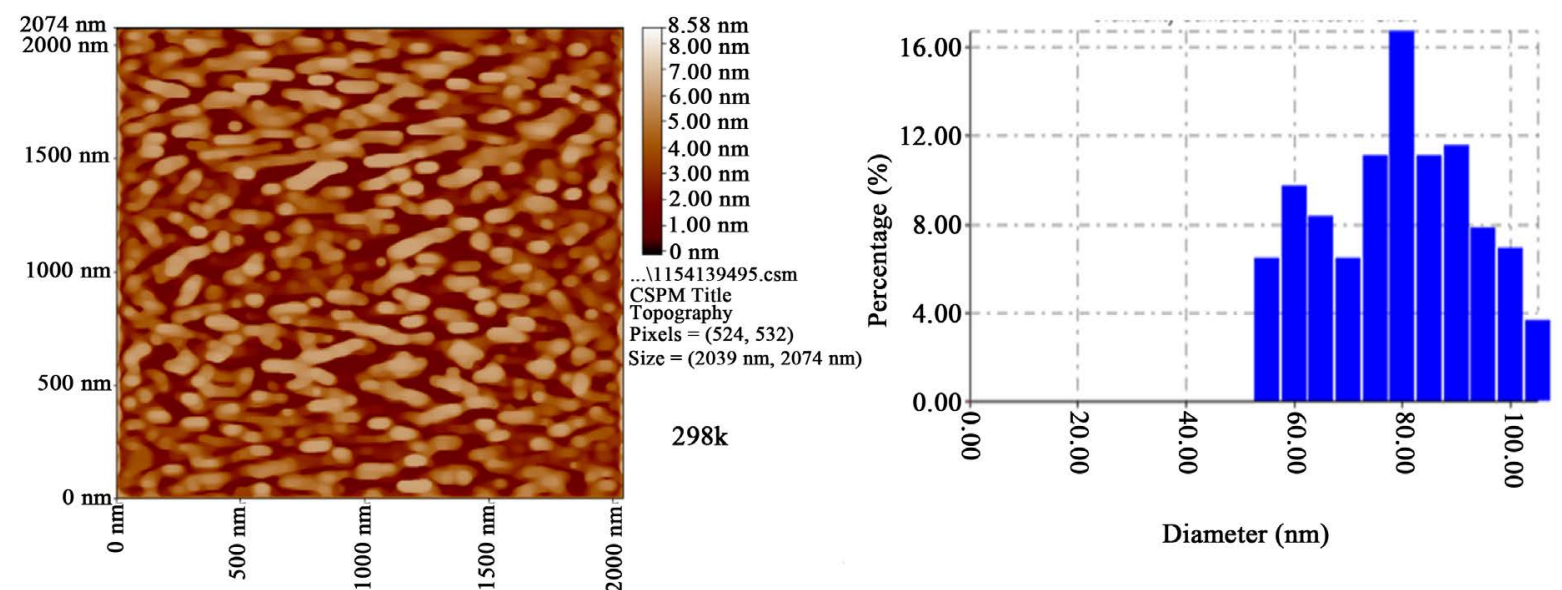

Diameter (nm)
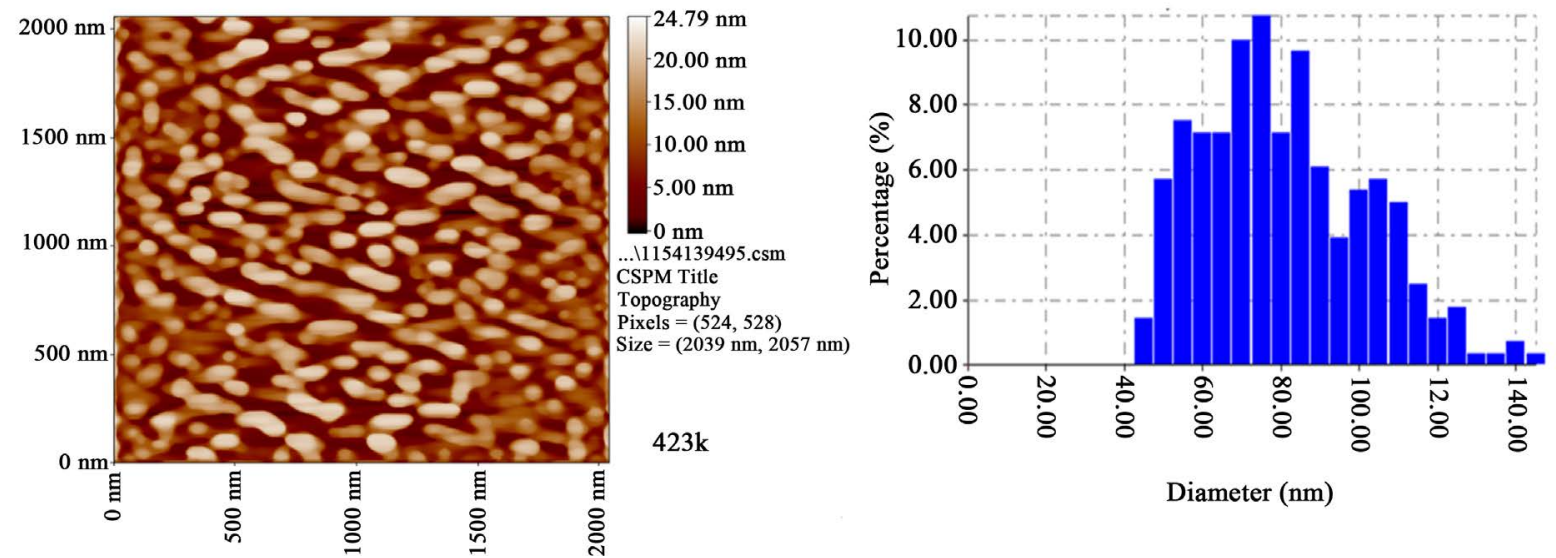

Figure 8. 2-D AFM images of CuPc thin films prepared by thermal evaporation method on glass substrate with granularity distribution charts at annealing temperatures (298, $432 \mathrm{~K})$. 

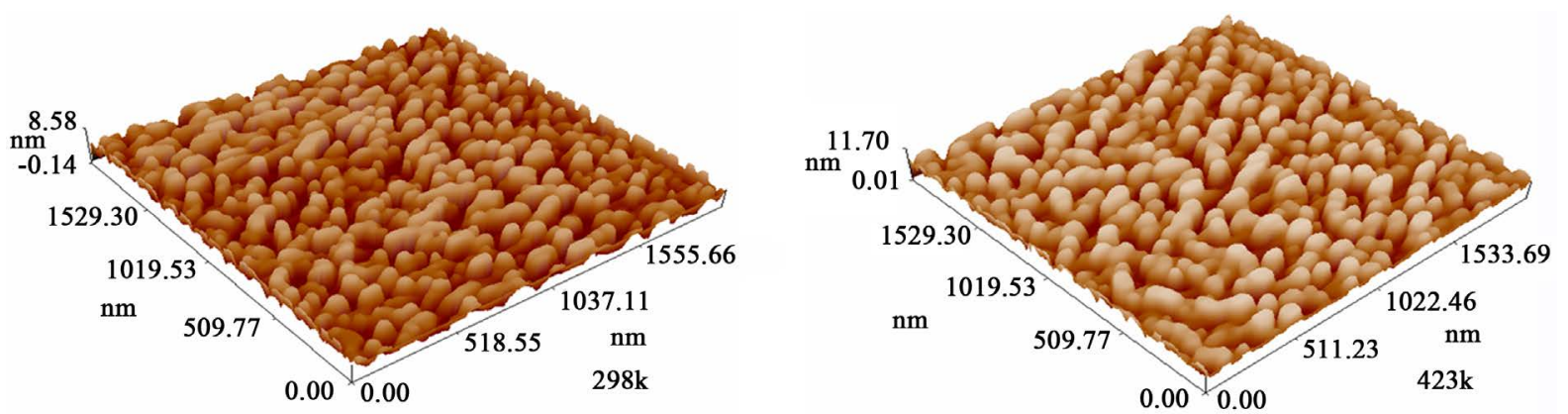

Figure 9. 3-D AFM images of CuPc thin films prepared by thermal evaporation method on glass substrate at annealing temperatures $(298,423 \mathrm{~K})$.

Table 3. This table shows the structure parameters of CuPc thin films prepared using PLD technique.

\begin{tabular}{cccccc}
\hline No. of shoots & $(\mathrm{hkl})$ & $2 \theta$ (degree) & $\mathrm{d}(\AA)$ & FWHM (degree) & $\mathrm{D}(\mathrm{nm})$ \\
\hline 150 & 100 & 7.1014 & 12.43789 & 0.55210 & 15.06 \\
400 & 100 & 7.1357 & 12.37818 & 1.39500 & 5.96 \\
\hline
\end{tabular}

Table 4. This table shows the grain size and roughness of CuPc thin films.

\begin{tabular}{ccc}
\hline $\mathrm{T}_{\mathrm{a}}(\mathrm{K})$ & Roughness average $(\mathrm{nm})$ & $\mathrm{D}(\mathrm{nm})$ \\
\hline 298 & 1.43 & 76.63 \\
323 & 1.33 & 64.60 \\
348 & 1.17 & 87.48 \\
373 & 5.29 & 78.43 \\
423 & 1.79 & 84.60 \\
\hline
\end{tabular}

mean square (rms) roughness of the film at different annealing temperatures $T_{a}$ are shown in Table 4, which instability with increasing $T_{a}$. The beast results can be seen for the film prepared at annealing temperature of $423 \mathrm{k}$.

The grain size and Roughness Average, represented as a function of annealing temperature are depicted in Figure 10 and Figure 11, respectively. The results show a fluctuation in grain size and Average Roughness with increasing annealing temperature.

Figure 12 and Figure 13 show AFM images of CuPc thin films prepared by PLD method at room temperature for $(150,400)$ shoots. It shows that the morphology of the CuPc thin films has large number of grain size and can be seen needle-like features for sample of (400) shoots, but for sample of (150) shoots cannot be seen that, which indicates the crystalline nature of the film. Planer terraces on the needles are also visible [17]. The surface roughness average for films prepared by PLD is about $(0.55,1.48) \mathrm{nm}$ and the Root Mean Square about $(0.64,1.74) \mathrm{nm}$ for $(150,400)$ shoots respectively, while the grain analysis report which estimated for CuPc thin films and the average diameter of the particles about 72.58 for 150 shoots and about $85.19 \mathrm{~nm}$ for 400 shoots.

\subsection{SEM Studies}

The Scanning Electron Microscopy (SEM) images of CuPc powder is shown in Figure 14(a), for comparison, a similar sample (CuPc thin film) was prepared by PLD and thermal evaporation methods and the SEM of these samples are shown in Figure 14(b) and Figure 14(c). It is easily observed that the samples prepared by thermal evaporation and PLD contains a pore while that CuPc powder contains stick structures with no pores at all.

\section{Conclusions}

Thin films of CuPc were fabricated by the thermal evaporation and PLD techniques on glass and silicon substrates with thickness $600 \mathrm{~nm}$ prepared at different annealing temperatures successfully. The outcome of this in- 


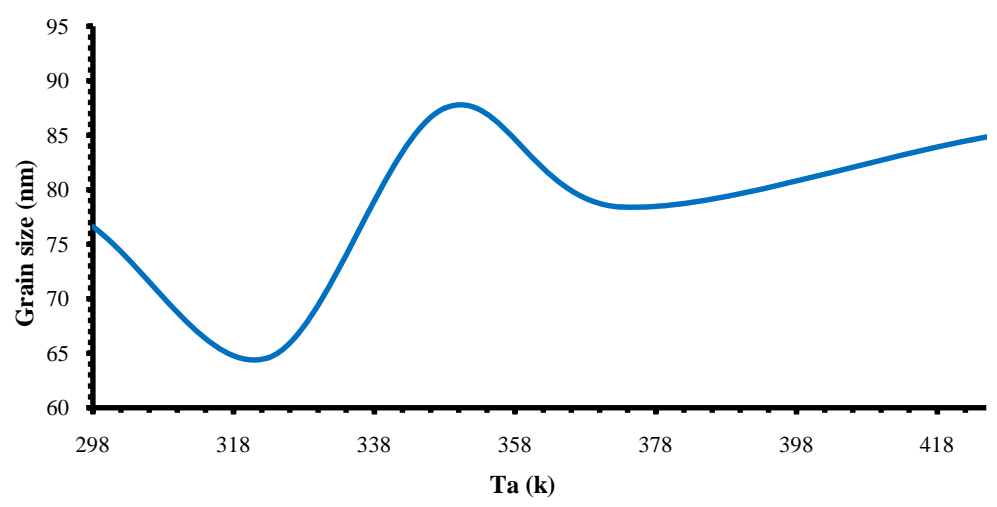

Figure 10. The grain size as a function of annealing temperature $\left(\mathrm{T}_{\mathrm{a}}\right)$ for CuPc thin film.

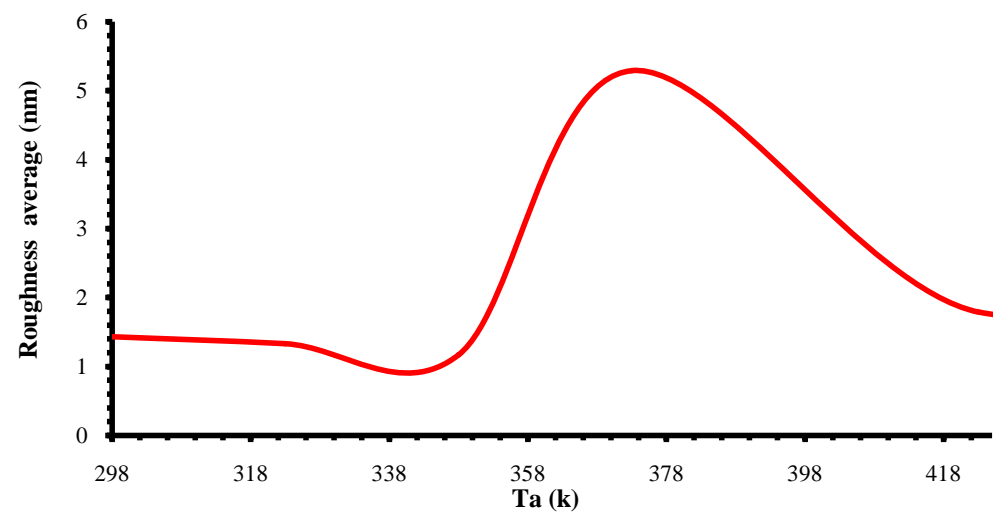

Figure 11. The roughness average as a function of annealing temperature $\left(\mathrm{T}_{\mathrm{a}}\right)$ for CuPc thin film.
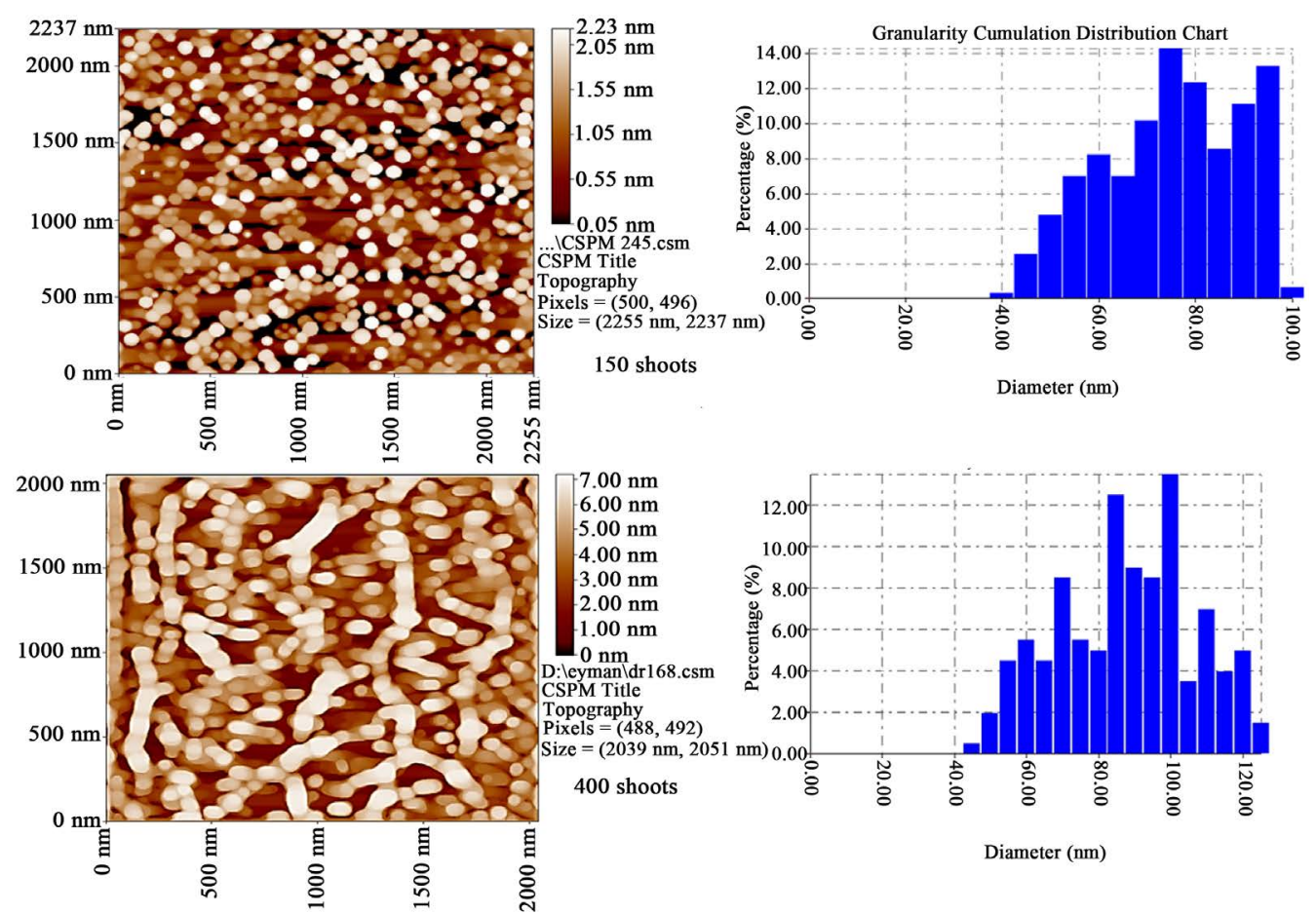

Figure 12. 2-D AFM images of CuPc thin films prepared by PLD method on glass substrate with Granularity Distribution charts for $(150,400)$ shoots at room temperature. 

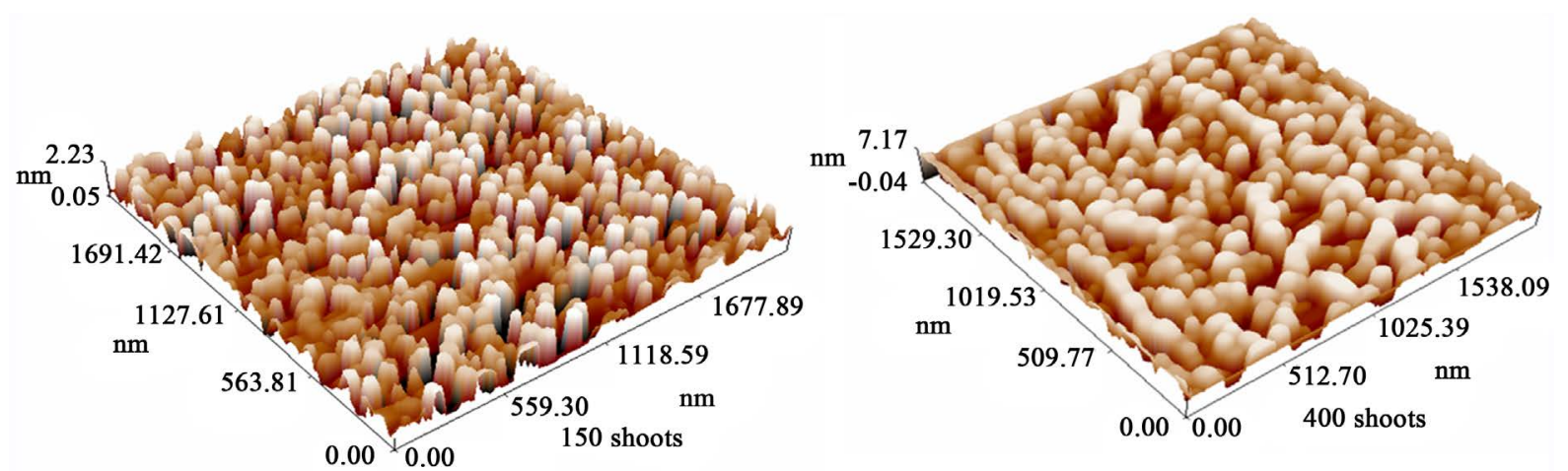

Figure 13. 3-D AFM images of CuPc thin films prepared by PLD ration method on glass substrate for $(150$, 400) shoots at room temperature.

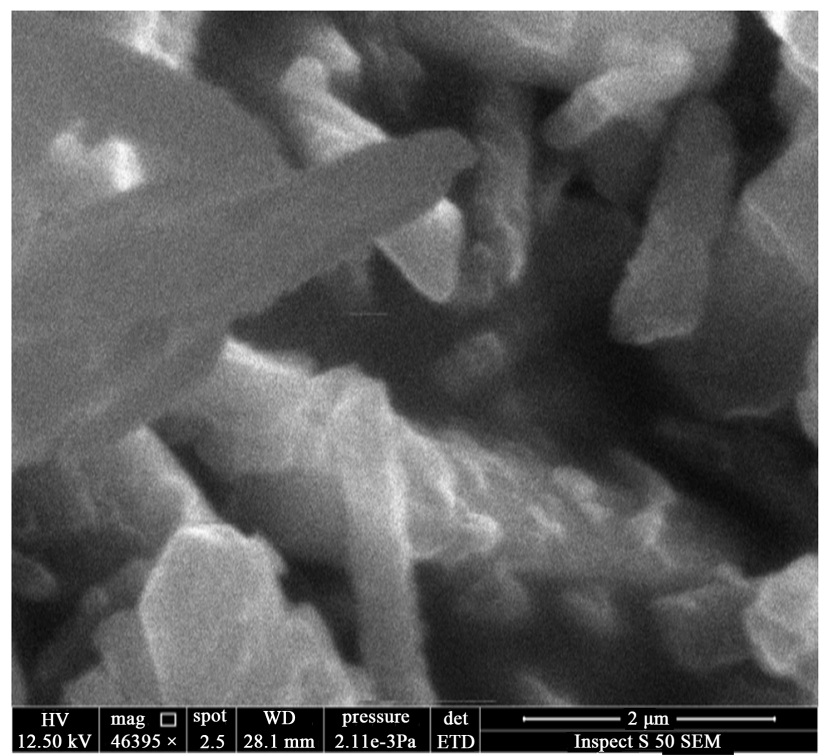

(a)

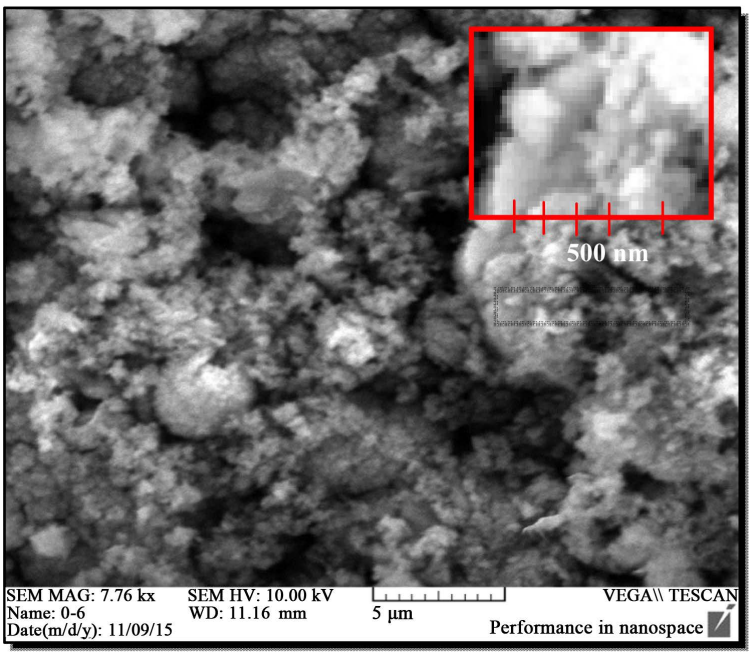

(b)

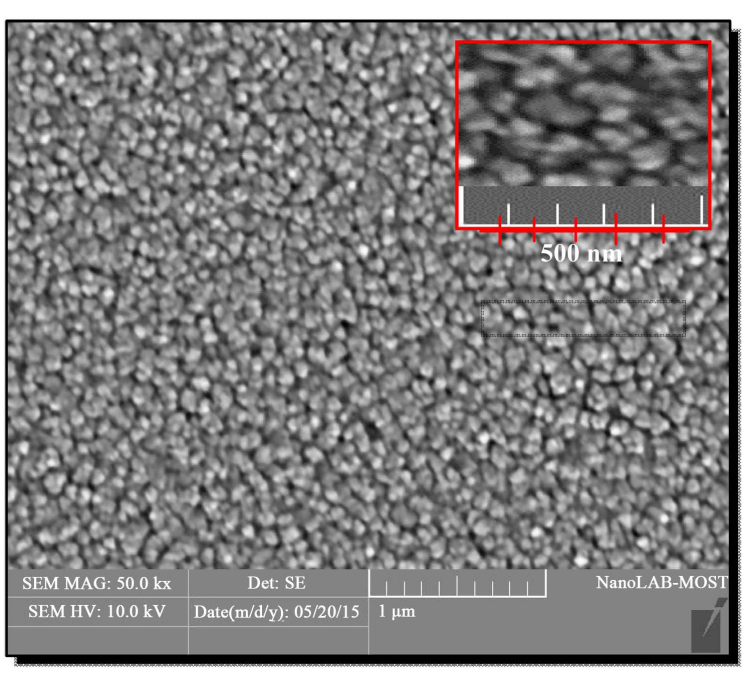

(c)

Figure 14. (a) Scanning Electron Microscope (SEM) of CuPc powder; (b) Scanning Electron Microscope (SEM) of CuPc thin film prepared by PLD; (c) Scanning Electron Microscope (SEM) of CuPc thin film prepared by thermal evaporation. 
vestigation can be summarized as follows:

- X-ray diffraction patterns obtained for CuPc powder showed $\alpha$-polycrystalline phase with monoclinic system, while the thin film of CuPc showed that it has single crystalline $\beta$-phase film oriented preferentially (100) plane as tetragonal. It can be seen that the intensity, $\mathrm{d}$ value and sharping of the main peak increase with annealing temperature, which shows crystallization of the films when annealing temperature increases.

- Diffraction peaks obtained for films prepared at higher annealing temperatures are sharp and that is due to the increased crysttalinity.

- The full width at half maximum (FWHM) intensity of preferential orientation shows that the FWHM is reduced with increasing annealing temperatures for temperature $>423 \mathrm{~K}$.

- The grain size for thin films prepared by thermal evaporation fluctuation from 19.58 - $20.94 \mathrm{~nm}$ for annealing temperatures 298 - $423 \mathrm{~K}$, this can be attributed to the onset of phase transition occurring in CuPc. Also this may be attributed to a phase transition or to the regrowth of phthalocyanine. This means that the crystallization of the films is a function of annealing temperature.

- The grain size for PLD films decreases with the increase of the number of shoots. This means that the crystallization of the films is a function of film thickness.

- By analyzing the AFM topographic images for thermal evaporation films, we found that there is a significant influence of annealing temperatures on the surface morphology of CuPc thin films, the crystal morphology and molecular orientation change with the annealing temperature and the grain size increases with increasing of annealing temperature.

- The surface roughness of CuPc thin films are changed with increasing annealing temperatures, which makes it useful for higher efficiency devices.

- We observed the homogeneous crystalline of CuPc thin film through SEM experiments.

\section{References}

[1] Hussein, M.T., Hassan, E.K. and Abdullah, E.T. (2015) Study the High Performance of Organic Semiconductor CuPc Field Effect Transistor. International Journal of Current Engineering and Technology, 5, 1593-1596.

[2] Hussein, M.T., Naji, I.S., Abdulameer, A.F., Hassen, E.K. and Badri, M.S. (2015) Capacitive-Resistive Measurements of Cobalt-Phthalocyanine Organic Humidity Sensors. Photonic Sensors, 5, 257-262. http://dx.doi.org/10.1007/s13320-015-0257-9

[3] Unni, K.N.N. and Menon, C.S. (2000) Electrical, Optical and Structural Studies on Nickel Phthalocyanine Thin Films, Materials Letters, 45, 326-330. http://dx.doi.org/10.1016/S0167-577X(00)00127-0

[4] El-Nahass, M.M., Bahabri, F.S., AL Ghamdi, A.A. and Al-Harbi, S.R. (2002) Structural and Transport Properties of Copper Phthalocyanine (CuPc) Thin Films. Egyptian Journal of Solids, 25, 307-321.

[5] Hussein, M.T. (2011) Study $\beta$-Phase Zn-Phthalocyanine Nanostructure by High Accuracy Preparation with Sublimation Method. International Journal of Science and Advanced Technology, 1, 137.

[6] Sidorov, A.N. and Kotlyar, I.P. (1961) Infrared Spectra of Phthalocyanines. I. The Effect of Crystalline Structure and of the Central Metallic Atom on the Phthalocyanine Molecule in the Solid State. Optics and Spectroscopy, 11, No. 92.

[7] Hussein, M.T., Nasir, E.M. and Al-Aarajiy, A.H. (2013) Structural and Surface Morphology Analysis of Nickel Phthalocyanine Thin Films. Advances in Materials Physics and Chemistry, 3, 113-119.

http://dx.doi.org/10.4236/ampc.2013.31A014

[8] Fedoruk, G.G., Sagaidak, D.I., Misevich, A.V. and Pochtenny, A.E. (1998) Electrical and Gas Sensing Properties of Copper Phthalocyanine-Polymer Composites. Sensors and Actuators B, 48, 351-355. http://dx.doi.org/10.1016/S0925-4005(98)00070-7

[9] Chu, C.-W., Shrotriya, V., Li, G. and Yang, Y. (2006) Tuning Acceptor Energy Level for Efficient Charge Collection in Copper-Phthalocyanine-Based Organic Solar Cells. Applied Physics Letters, 88, Article ID: 153504. http://dx.doi.org/10.1063/1.2194207

[10] Eason, R. (2007) Pulsed Laser Deposition of Thin Films Applications-Led Growth of Functional Materials. John Wiley \& Sons, Inc., UK.

[11] Aziza, F., Sulaimana, K., Muhammad, M.R., Sayyaad, M.H. and Karimov, Kh. (2011) Influence of Thermal Annealing on the Structural Properties of Vanadyl Phthalocyanine Thin Films: A Comparative Study, World Academy of Science. Engineering and Technology, 56, 852-854.

[12] Gu, D., Chen, Q.Y., Shu, J.P., Tang, X.D., Gan, F.X., Shenb, S.Y., Liu, K. and Xu, H.J. (1995) Optical Recording Performance of Thin Films of Phthalocyanine Compounds. Thin Solid Films, 257, 88-93. 
http://dx.doi.org/10.1016/0040-6090(94)06327-3

[13] Shihub, S. and Gould, R. (1993) Structure and Phase-Change Phenomena in Evaporated Thin Films of Cobalt Phthalocyanine. Physica Status Solidi A, 139, 129-138. http://dx.doi.org/10.1002/pssa.2211390110

[14] Nonaka, T., Nakagawa, Y., Mori, Y., Hirai, M., Matsunobe, T., Nakamura, M., Takahagi, T. and Ishitani, A. (1995) Epitaxial Growth of $\alpha$-Copper Phthalocyanine Crystal on si(001) Substrate by Organic Molecular Beam Deposition. Thin Solid Films, 256, 262-267. http://dx.doi.org/10.1016/0040-6090(94)06304-4

[15] Kwak, T.H., Kang, H.S., Kim, K., Cho, M.Y., Lee, J.W. and Joo, J. (1995) Copper-Phthalocyanine Based Organic Thin Film Transistor. NMP, 54, 630-631.

[16] Hassan, A.K. and Gould (1992) Structural Studies of Thermally Evaporated Thin Films of Copper Phthalocyanine. Physica Status Solidi (a), 132, 91. http://dx.doi.org/10.1002/pssa.2211320110

[17] Della Pirriera, M., Puigdollers, J., Voz, C., Stella, M., Bertomeu, J. and Alcubilla, R. (2009) Optoelectronic Properties of CuPc Thin Films Deposited at Different Substrate Temperatures. Applied Physics, $42,5$. http://dx.doi.org/10.1088/0022-3727/42/14/145102 